\title{
Research on the digital sensor based on STM32 for leakage current
}

\author{
YUAN Kai-hong \\ (Hunan Railway Professional Technology College, ZhuZhou Hunan 412001)
}

Keywords: STM32; CAN bus; leakage current; sensor; digital processing

\begin{abstract}
STM32 platform is developed. It is in a high precision simulation based on leakage current sensor by digitization and improvement, using CAN bus for data communication, real-time display clear, has the ability to meet the independent and comprehensive treatment of treatment with multiple other the same sensor, accurate detection, response fast, safe and reliable data communication lines. Is a kind of current advanced digital sensor for leakage current.
\end{abstract}

\section{Introduction}

Electrical safety problems are becoming more and more important with the railway electrification and a large number of electrical equipment and facilities on the locomotive. The insulation and leakage is an important indicator to monitor the electrical safety, through the strict implementation and relevant technical requirements of insulation, leakage, electrical defects can effectively monitor the power supply equipment, prevent leakage, short-circuit caused casualties and fire accidents. In order to ensure the electrical safety of locomotive, passenger car, real-time monitoring of leakage protection alarm and grounding protection technology, the leakage current of locomotive power supply bus and real-time monitoring of the situation, in case of leakage current or exceed the standard grounding protection action, stop the power output. At the same time, the former Ministry of Railways to wire and cable, electrical facilities on the locomotive insulation has made it clear that the requirements for testing in the depot and ensure delivery of good quality.

The leakage current sensor of the embedded system is realized to detect the leakage current in the locomotive, and finally realize the automatic control. The most important feature is that it can detect and transform quickly and accurately, and transfer the digital information, which can be used for the analysis and processing of computer system. Foreign embedded system sensors used earlier, the technology is more mature, but due to the wide range of sensor applications, different requirements, strong market demand. The development of the CAN bus on board leakage current sensor based on STM32 platform is developed in this background. CAN bus mobile STM32 platform leakage current sensor is developed based on the simulation of the most advanced leakage current sensor to realize digital transformation, digital communication and the line number less, safety, high reliability CAN bus. Can be convenient to achieve multi-point detection centralized processing.

\section{System principle}

This paper mainly studies the STM32 platform based on CAN bus digital leakage current detection sensor, leakage detection device solves the hardware system using the original locomotive block interface line, too much, the anti-interference ability is not strong, the problem of high failure rate. STM32 platform CAN bus digital leakage current detection sensor is connected through the CAN bus based communication lines connected in series together, greatly reduce the line number; at the same time the conversion of the A/D STM32 processor, reduce internal sensor hardware block.

CAN bus leakage current detection digital sensor based on STM32 platform mainly detect parameters: the detection of leakage current range is $0 \sim 300 \mathrm{~mA}$, the detection accuracy is $1 \%$, the linearity is $1 \%$. 


\section{Hardware system design}

STM32 is a micro controller based on CORTEX-M3 kernel, high performance, low cost and low power consumption. Its clock frequency of $72 \mathrm{MHz}$, to achieve high-end computing. Embedded FLASH 512KB program memory. Rich peripherals, ART, SPI and other serial interface as well as the maximum turnover rate of 18 PIO MHz. More important is that it has the fastest 1 TXS conversion speed of the dual 12 bit precision ADC, this fast acquisition, high performance of ADC is very suitable for the rapid acquisition and rapid processing of data, and the internal integration of dual CAN controller. It is only the CAN protocol V2.0A and V2.0B, the baud rate is up to $1 \mathrm{MB} / \mathrm{S}$, with 3 transmit email and 23 depth FIFO, with minimal C PU load to receive a large number of efficient packet processing. Based on the function of the appeal, so choose TM32 S as the main controller design.

CAN is the controller area network (Area Network Controller, CAN) for short, is developed by the German BOSCH company, and eventually become an international standard. Is one of the most widely used field bus in the world. In recent years, it has high reliability and good error detection ability. It is widely used in the automobile computer control system and the environment temperature is bad, the electromagnetic radiation is strong and the vibration of the industrial environment.

Leakage current tester pre purchase with CAN bus interface STM32 development board, so as to save the development cycle, and reduce the risk of early development. After the development board up through CAN communication, and then according to the actual size of the analog sensor, the installation of a small data processing unit. Complete analog signal acquisition and CAN communication.

The whole communication frame is carried out by the host in real-time to poll each sub module. Each sub module has its own separate communication address to ensure that the data is not in conflict. Using CAN bus to transmit the information to avoid the faults caused by too much lead. CAN bus is a standard embedded industrial control network, has high reliability and good error detection ability of attention, is widely used in harsh environment temperature, strong electromagnetic radiation and vibration of large industrial environment.

\section{Device and experiment}

The working principle of the system is in the boot device to collect train leakage current value, according to a single locomotive $4.7 \mathrm{~mA}$ as the benchmark for judging train number as a device to determine whether the reference data leakage current; when the locomotive power supply system has a grounding device, current detection sensor detects the current signal at the same time, record and display, then according to the set locomotive leakage current alarm value and the detected leakage current value, if beyond recognition device will produce ground vehicle alarm and a display lamp; the recording time, leakage fault grounding flow value at the same time identification device, note indicating the grounding fault in the state of the locomotive.

Experimental conditions: ambient temperature: room temperature. Experimental equipment: adjustable switching power supply (0-5A 0-150V), a large power wire wound resistor (40/1500W), a leakage current sensor, a leakage current detector mainframe, a number of wires. Experimental analysis of two cases.

(1)DC positive and negative wire (double line) all through the sensor simulation of leakage test, the wiring principle as shown in Fig.1.

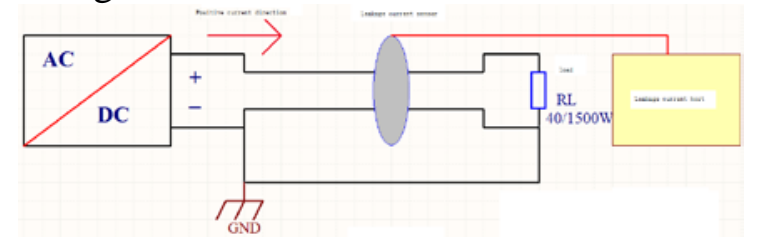

Fig.1. Wiring diagram of two line detection experiment 
The experimental data are shown in tab. 1 .

Tab. 1 experimental record form (double line)

\begin{tabular}{|c|c|c|c|}
\hline $\begin{array}{c}\text { Serial } \\
\text { number }\end{array}$ & Voltage $(\mathrm{V})$ & $\begin{array}{c}\text { Switching power } \\
\text { supply current }(\mathrm{mA})\end{array}$ & $\begin{array}{c}\text { Instrument display leakage } \\
\text { current }(\mathrm{mA})\end{array}$ \\
\hline 1 & 0.0 & 0.0 & 0.0 \\
\hline 2 & 1.0 & 20 & 20 \\
\hline 3 & 1.5 & 30 & $29 \sim 31$ \\
\hline 4 & 2.0 & 40 & $41 \sim 42$ \\
\hline 5 & 2.5 & 60 & $59 \sim 60$ \\
\hline 6 & 3.0 & 80 & 79 \\
\hline 7 & 3.5 & 90 & 91 \\
\hline 8 & 4.0 & 100 & $99 \sim 101$ \\
\hline 9 & 4.5 & 110 & 109 \\
\hline 10 & 5.0 & 120 & 120 \\
\hline 11 & 5.5 & 130 & 148 \\
\hline 12 & 6.0 & 150 & $160 \sim 162$ \\
\hline 13 & 6.5 & 160 & $170 \sim 181$ \\
\hline 14 & 7.0 & 170 & $200 \sim 201$ \\
\hline 15 & 7.5 & 180 & 211 \\
\hline 16 & 8.0 & 200 & $220 \sim 221$ \\
\hline 17 & 8.5 & 210 & $229 \sim 230$ \\
\hline 18 & 9.0 & 220 & 249 \\
\hline 19 & 9.5 & 230 & 250 \\
\hline 20 & 10 & 250 & 180 \\
\hline
\end{tabular}

(2) Only the DC line (single) simulation experiment through the leakage of the sensor, the wiring principle diagram as shown in Fig 2.

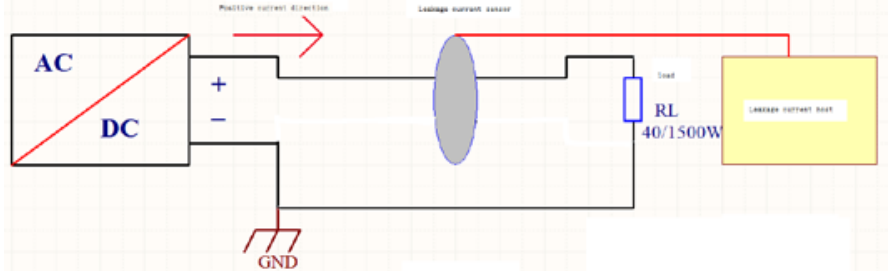

Fig 2. The original single line diagram

The experimental data are shown in Tab.2.

Tab. 2 experimental record form (single line)

\begin{tabular}{|c|c|c|c|}
\hline $\begin{array}{c}\text { Serial } \\
\text { number }\end{array}$ & Voltage (V) & $\begin{array}{c}\text { Switching power supply current } \\
(\mathrm{mA})\end{array}$ & $\begin{array}{c}\text { Instrument display leakage } \\
\text { current (mA) }\end{array}$ \\
\hline 1 & 0.5 & 10 & 12 \\
\hline 2 & 1.0 & 20 & 22 \\
\hline 3 & 1.5 & 40 & 38 \\
\hline 4 & 2.0 & 50 & 49 \\
\hline 5 & 2.5 & 60 & 62 \\
\hline 6 & 3.0 & 70 & 71 \\
\hline 7 & 3.5 & 90 & 89 \\
\hline 8 & 4.0 & 100 & 102 \\
\hline 9 & 4.5 & 120 & 121 \\
\hline 10 & 5.5 & 130 & 129 \\
\hline 11 & 6.0 & 150 & 151 \\
\hline 12 & 6.5 & 160 & 160 \\
\hline 13 & 7.0 & 170 & 172 \\
\hline 14 & 7.5 & 180 & 179 \\
\hline 15 & 8.0 & 200 & 201 \\
\hline 16 & 8.5 & 210 & 209 \\
\hline 17 & 9.0 & 220 & 221 \\
\hline 18 & 9.5 & 240 & 240 \\
\hline 19 & 10 & 250 & 253 \\
\hline
\end{tabular}




\section{Conclusion}

Through the analysis of the principle of leakage project design of digital current sensor, hardware design and software design, and then through the actual leakage current detection, data show that the detection of preparation, high precision, convenient detection, the digital leakage current sensor has good application value.

Fund support: Science research project of Hunan Provincial Department of Education(14C0756)

--- Development of a CAN bus based on STM32 bus in vehicle leakage current detection digital sensor

\section{References}

[1] Wang Da-li. Hybrid braking system of electric vehicle based on CAN bus [J]. Instrument technique and sensor. 2013(11): 108-110

[2]Yuan Kai-hong.Wei Li-jun.Tang Dong-mei. CAN bus on board leakage current digital sensor based on STM32 platform [J]. Sensors and micro systems. 2013, 33(3): 118-120.

[3]Tang Ya-ping. Wei Li-jun.Zhang Min-san. Research and application of leakage current digital sensor for locomotive [J]. Sensors and micro systems. 2014，33(12): 45-47.

[4] Chen Zhi-wang. 《STM32 embedded microcontroller principle, application technology》[M]. Electronic Industry Publishing House. 2012 年

[5] Ma Zhong-mei, Ji Shun-xin. 《C language application program design of single chip microcomputer》[M]. Beihang University press.2003.132 147

[6] Wu Xiao-feng. 《 Manual of the function and application example of computer integrated circuit》[M]. People's Posts and Telecommunications Press.1997.291 335 